\title{
The stability of bi-additive functional equation on a restricted domain
}

\author{
Weiwei Liu, a , Peisheng $\mathrm{Ji}^{1, \mathrm{~b}}$ and Lin Wang ${ }^{2, \mathrm{c}}$ \\ ${ }^{1}$ College of Mathematics, QingDao University, QingDao 266071, PR China \\ ${ }^{2}$ College of Mathematics and physics, QingDao University of Science \& \\ Technology, QingDao 266071, PR China \\ aliuww1229@163.com, bjipeish@yahoo.com, wlzwl@163.com
}

Keywords: Hyers-Ulam stability problem, restricted domain, bi-additive functional equation Abstract. The stability problem of the bi-additive functional equation $f\left(x_{11}+x_{12}, x_{21}+x_{22}\right)=\sum_{i_{1}, i_{2} \in\{1,2\}} f\left(x_{1 i_{1}}, x_{2 i_{2}}\right)$ has been studied by Krzysztof Ciepliński. In this paper, we will investigate the stability of the bi-additive functional equation on a restricted domain.

\section{Introduction}

The stability problem of functional equations originated from the stability problem of group homomorphisms which was presented by S.M. Ulam [1] in 1940. In 1941, Hyers [2] became the first mathematician to give an affirmative partial answer to this question. In 1978, Th.M. Rassias [3] took account of the unbounded Cauchy difference in Hyers' theorem and then obtained the results for linear mappings. The result of Rassias has played an important role in the development of the Hyers-Ulam-Rassias stability phenomenon. Since then numbers of papers on the stability problem for various function equations have been published (See [4,5] and references therein).

Throughout this paper, let $X$ be a complex normed space and let $Y$ be a complex Banach space.

Definition 1.1. A mapping $f: X^{2} \rightarrow Y$ is called a bi-additive mapping if $f$ satisfies the system of equations

$$
\begin{aligned}
& f\left(x_{11}+x_{12}, x_{21}\right)=f\left(x_{11}, x_{21}\right)+f\left(x_{12}, x_{21}\right), \\
& f\left(x_{11}, x_{21}+x_{22}\right)=f\left(x_{11}, x_{21}\right)+f\left(x_{11}, x_{22}\right), \\
& x_{11}, x_{12}, x_{21}, x_{22} \in X .
\end{aligned}
$$

Lemma 1.1. A function $f: X^{2} \rightarrow Y$ satisfies (1.1) if and only if it satisfies the functional equation

$$
f\left(x_{11}+x_{12}, x_{21}+x_{22}\right)=\sum_{i_{1}, i_{2} \in\{1,2\}} f\left(x_{1 i_{1}}, x_{2 i_{2}}\right), x_{11}, x_{12}, x_{21}, x_{22} \in X .
$$

Krzysztof Ciepliński [6] contributed to the stability problem of the multi-additive functional equation, when $n=2$ we give the following theorem:

Theorem 1.1. ${ }^{[6]}$ Let $V$ be a commutative semigroup with an identity element and $W$ be a Banach space. Assume also that $\varphi: V^{4} \rightarrow[0, \infty)$ is a mapping such that

$$
\Psi\left(x_{11}, x_{12}, x_{21}, x_{22}\right)=\sum_{n=0}^{\infty} \frac{1}{4^{(n+1)}} \varphi\left(2^{n} x_{11}, 2^{n} x_{12}, 2^{n} x_{21}, 2^{n} x_{22}\right)<\infty,\left(x_{11}, x_{12}, x_{21}, x_{22}\right) \in V^{4} .
$$

If $f: V^{2} \rightarrow W$ is a function satisfying

$$
\left\|f\left(x_{11}+x_{12}, x_{21}+x_{22}\right)-\sum_{i_{1}, i_{2} \in\{1,2\}} f\left(x_{1 i_{1}}, x_{2 i_{2}}\right)\right\| \leq \varphi\left(x_{11}, x_{12}, x_{21}, x_{22}\right),
$$

for all $\left(x_{11}, x_{12}, x_{21}, x_{22}\right) \in V^{4}$, then there exist a unique bi-additive mapping $F: V^{2} \rightarrow W$ such that

$$
\left\|f\left(x_{11}, x_{21}\right)-F\left(x_{11}, x_{21}\right)\right\| \leq \Psi\left(x_{11}, x_{11}, x_{21}, x_{21}\right),\left(x_{11}, x_{21}\right) \in V^{2} \text {. }
$$

The function $F$ is given by

$$
F\left(x_{11}, x_{21}\right)=\lim _{n \rightarrow \infty} \frac{1}{4^{n}} f\left(2^{n} x_{11}, 2^{n} x_{21}\right),\left(x_{11}, x_{21}\right) \in V^{2} .
$$

In 1994, F. Skof [7] proved an important result on Hyers-Ulam-Rassias stability of functional equation on a restricted domain. In 1998 S.M. Jung [8] investigated the Hyers-Ulam stability of 
additive and quadratic mappings on restricted domains. The next year, Bogdan Batko and Jacek Tabor [9] proved the result on the stability of an alternative Cauchy equation on a restricted domain. In 2013, Yang-Hi Lee [10] solved the stability problem for the quadratic-additive type functional equation on a restricted domain. In this paper, we will investigate the stability of the bi-additive functional equation on a restricted domain.

\section{Main results}

Theorem 2.1. Let $d>0$ and $\delta \geq 0$ be given. Assume that a function $f: X^{2} \rightarrow Y$ satisfies the inequality

$$
\left\|f\left(x_{11}+x_{12}, x_{21}+x_{22}\right)-\sum_{i_{1}, i_{2} \in\{1,2\}} f\left(x_{1 i_{1}}, x_{2 i_{2}}\right)\right\| \leq \delta,
$$

for all $x_{11}, x_{12}, x_{21}, x_{22} \in X$ with $\left\|x_{11}\right\|+\left\|x_{12}\right\| \geq d$ and $\left\|x_{21}\right\|+\left\|x_{22}\right\| \geq d$. Then there exists a unique bi-additive function $F: X^{2} \rightarrow Y$ such that

$$
\left\|F\left(x_{1}, x_{2}\right)-f\left(x_{1}, x_{2}\right)\right\| \leq \frac{25}{3} \delta
$$

for all $x_{1}, x_{2} \in X$.

Proof: Assume $\left\|x_{11}\right\|+\left\|x_{12}\right\|<d$ and $\left\|x_{21}\right\|+\left\|x_{22}\right\| \geq d$. If $x_{11}=x_{12}=0$, then we choose a $p_{1} \in X \quad$ with $\quad\left\|p_{1}\right\|=d$. Otherwise, let $p_{1}=\left(1+d /\left\|x_{11}\right\|\right) x_{11}$ for $\left\|x_{11}\right\| \geq\left\|x_{12}\right\|$ or $p_{1}=\left(1+d /\left\|x_{12}\right\|\right) x_{12}$ for $\left\|x_{11}\right\|<\left\|x_{12}\right\|$. Clearly, we see

$$
\begin{aligned}
& \left\|x_{11}+x_{12}-2 p_{1}\right\|+\left\|2 p_{1}\right\| \geq d \\
& \left\|x_{11}+x_{12}-2 p_{1}\right\|+\left\|p_{1}\right\| \geq d \\
& \left\|x_{11}\right\|+\left\|x_{12}-p_{1}\right\| \geq d \\
& \left\|x_{12}-p_{1}\right\|+\left\|2 p_{1}\right\| \geq d \\
& \left\|x_{12}\right\|+\left\|p_{1}\right\| \geq d
\end{aligned}
$$

From (2.1), (2.3), and the relation

$$
\begin{aligned}
& f\left(x_{11}+x_{12}, x_{21}+x_{22}\right)-\sum_{i_{1}, i_{2} \in\{1,2\}} f\left(x_{1 i_{1}}, x_{2 i_{2}}\right) \\
& =f\left(x_{11}+x_{12}, x_{21}+x_{22}\right)-f\left(x_{11}+x_{12}-2 p_{1}, x_{21}\right) \\
& -f\left(x_{11}+x_{12}-2 p_{1}, x_{22}\right)-f\left(2 p_{1}, x_{21}\right)-f\left(2 p_{1}, x_{22}\right) \\
& -\left[f\left(x_{11}+x_{12}-p_{1}, x_{21}+x_{22}\right)-f\left(x_{11}+x_{12}-2 p_{1}, x_{21}\right)\right. \\
& \left.-f\left(x_{11}+x_{12}-2 p_{1}, x_{22}\right)-f\left(p_{1}, x_{21}\right)-f\left(p_{1}, x_{22}\right)\right] \\
& +f\left(x_{11}+x_{12}-p_{1}, x_{21}+x_{22}\right)-f\left(x_{11}, x_{21}\right)-f\left(x_{11}, x_{22}\right) \\
& -f\left(x_{12}-p_{1}, x_{21}\right)-f\left(x_{12}-p_{1}, x_{22}\right) \\
& -\left[f\left(x_{12}+p_{1}, x_{21}+x_{22}\right)-f\left(2 p_{1}, x_{21}\right)-f\left(2 p_{1}, x_{22}\right)\right. \\
& \left.-f\left(x_{12}-p_{1}, x_{21}\right)-f\left(x_{12}-p_{1}, x_{22}\right)\right] \\
& +f\left(x_{12}+p_{1}, x_{21}+x_{22}\right)-f\left(p_{1}, x_{21}\right) \\
& -f\left(p_{1}, x_{22}\right)-f\left(x_{12}, x_{21}\right)-f\left(x_{12}, x_{22}\right),
\end{aligned}
$$

we get

$$
\left\|f\left(x_{11}+x_{12}, x_{21}+x_{22}\right)-\sum_{i_{1}, i_{2} \in\{1,2\}} f\left(x_{1 i_{1}}, x_{2 i_{2}}\right)\right\| \leq 5 \delta .
$$

Thus the inequality (2.4) holds true for all $x_{11}, x_{12} \in X$ and $\left\|x_{21}\right\|+\left\|x_{22}\right\| \geq d$.

Assume $\left\|x_{21}\right\|+\left\|x_{22}\right\|<d$. If $x_{21}=x_{22}=0$, then we choose a $p_{2} \in X$ with $\left\|p_{2}\right\|=d$. Otherwise, let $p_{2}=\left(1+d /\left\|x_{21}\right\|\right) x_{21}$ for $\left\|x_{21}\right\| \geq\left\|x_{22}\right\|$ or $p_{2}=\left(1+d /\left\|x_{22}\right\|\right) x_{22}$ for $\left\|x_{21}\right\|<\left\|x_{22}\right\|$. Clearly, we see

$$
\begin{aligned}
& \left\|x_{21}+x_{22}-2 p_{2}\right\|+\left\|2 p_{2}\right\| \geq d, \\
& \left\|x_{21}+x_{22}-2 p_{2}\right\|+\left\|p_{2}\right\| \geq d, \\
& \left\|x_{21}\right\|+\left\|x_{22}-p_{2}\right\| \geq d,
\end{aligned}
$$


$\left\|x_{22}-p_{2}\right\|+\left\|2 p_{2}\right\| \geq d$,

$\left\|x_{22}\right\|+\left\|p_{2}\right\| \geq d$.

From (2.5), (2.4), and the relation

$$
\begin{aligned}
& f\left(x_{11}+x_{12}, x_{21}+x_{22}\right)-\sum_{i_{1}, i_{2} \in\{1,2\}} f\left(x_{1 i_{1}}, x_{2 i_{2}}\right) \\
= & f\left(x_{11}+x_{12}, x_{21}+x_{22}\right)-f\left(x_{11}, x_{21}+x_{22}-2 p_{2}\right) \\
- & f\left(x_{12}, x_{21}+x_{22}-2 p_{2}\right)-f\left(x_{11}, 2 p_{2}\right)-f\left(x_{12}, 2 p_{2}\right) \\
- & {\left[f\left(x_{11}+x_{12}, x_{21}+x_{22}-p_{2}\right)-f\left(x_{11}, x_{21}+x_{22}-2 p_{2}\right)\right.} \\
- & \left.f\left(x_{11}, p_{2}\right)-f\left(x_{12}, x_{21}+x_{22}-2 p_{2}\right)-f\left(x_{12}, p_{2}\right)\right] \\
+ & f\left(x_{11}+x_{12}, x_{21}+x_{22}-p_{2}\right)-f\left(x_{11}, x_{21}\right)-f\left(x_{11}, x_{22}-p_{2}\right) \\
- & f\left(x_{12}, x_{21}\right)-f\left(x_{12}, x_{22}-p_{2}\right) \\
- & {\left[f\left(x_{11}+x_{12}, x_{22}+p_{2}\right)-f\left(x_{11}, 2 p_{2}\right)-f\left(x_{11}, x_{22}-p_{2}\right)\right.} \\
- & \left.f\left(x_{12}, 2 p_{2}\right)-f\left(x_{12}, x_{22}-p_{2}\right)\right] \\
+ & f\left(x_{11}+x_{12}, x_{22}+p_{2}\right)-f\left(x_{11}, x_{22}\right) \\
- & f\left(x_{12}, x_{22}\right)-f\left(x_{11}, p_{2}\right)-f\left(x_{12}, p_{2}\right),
\end{aligned}
$$

we get

$$
\left\|f\left(x_{11}+x_{12}, x_{21}+x_{22}\right)-\sum_{i_{1}, i_{2} \in\{1,2\}} f\left(x_{1 i_{1}}, x_{2 i_{2}}\right)\right\| \leq 25 \delta .
$$

Thus the inequality (2.6) holds true for all $x_{11}, x_{12}, x_{21}, x_{22} \in X$.

Fix $\left(x_{11}, x_{12}\right) \in X^{2}$. Putting $x_{i 1}=x_{i 2}$ for $i \in\{1,2\}$ in (2.6) we get

$\left\|f\left(2 x_{11}, 2 x_{21}\right)-4 f\left(x_{11}, x_{21}\right)\right\| \leq 25 \delta$.

Dividing both sides of the above inequality by $4^{n+1}$ and replacing $x_{i 1}$ by $2^{n} x_{i 1}$ for $i \in\{1,2\}$ we see that $\left\|\frac{1}{4^{n+1}} f\left(2^{n+1} x_{11}, 2^{n+1} x_{21}\right)-\frac{1}{4^{n}} f\left(2^{n} x_{11}, 2^{n} x_{21}\right)\right\| \leq \frac{25}{4^{n+1}} \delta$,

and consequently for any non-negative integer $m$ with $m<n$ we obtain

$$
\begin{aligned}
& \left\|\frac{1}{4^{n}} f\left(2^{n} x_{11}, 2^{n} x_{21}\right)-\frac{1}{4^{m}} f\left(2^{m} x_{11}, 2^{m} x_{21}\right)\right\| \\
& \leq\left\|\frac{1}{4^{n}} f\left(2^{n} x_{11}, 2^{n} x_{21}\right)-\frac{1}{4^{n-1}} f\left(2^{n-1} x_{11}, 2^{n-1} x_{21}\right)\right\|+\mathrm{L} \\
& +\left\|\frac{1}{4^{m+1}} f\left(2^{m+1} x_{11}, 2^{m+1} x_{21}\right)-\frac{1}{4^{m}} f\left(2^{m} x_{11}, 2^{m} x_{21}\right)\right\|=\sum_{j=m}^{n-1} \frac{25}{4^{j+1}} \delta .
\end{aligned}
$$

Letting $m \rightarrow \infty$ in (2.7) we see that $\left\|\frac{1}{4^{n}} f\left(2^{n} x_{11}, 2^{n} x_{21}\right)-\frac{1}{4^{m}} f\left(2^{m} x_{11}, 2^{m} x_{21}\right)\right\| \rightarrow 0$. Hence, the Hyers-Ulam sequence $\left\{\frac{1}{4^{n}} f\left(2^{n} x_{11}, 2^{n} x_{21}\right)\right\}$ is a Cauchy sequence. Since the space $Y$ is complete, this sequence is convergent and we define $F: X^{2} \rightarrow Y$ by

$$
F\left(x_{11}, x_{21}\right)=\lim _{n \rightarrow \infty} \frac{1}{4^{n}} f\left(2^{n} x_{11}, 2^{n} x_{21}\right) \text {. }
$$

Putting $m=0$, letting $n \rightarrow \infty$ in (2.7) we see that (2.2) holds.

Next, fix also $\left(x_{11}, x_{12}\right) \in X^{2}$ and note that according to (2.6) we have

$$
\left\|\frac{1}{4^{n}} f\left(2^{n} x_{11}+2^{n} x_{12}, 2^{n} x_{21}+2^{n} x_{22}\right)-\frac{1}{4^{n}} \sum_{i_{1}, i_{2} \in\{1,2\}} f\left(2^{n} x_{1 i_{1}}, 2^{n} x_{2 i_{2}}\right)\right\| \leq \frac{25}{4^{n}} \delta \text {. }
$$

Letting $n \rightarrow \infty$ in the above inequality and using (2.8) we see that the mapping $F$ is a bi-additive mapping.

Finally, assume that $F^{\prime}: X^{2} \rightarrow Y$ is another bi-additive mapping satisfying (2.2) and fix $n \in N \cup\{0\}$. Then, using the bi-additivity of $F$ and $F^{\prime}$, we have

$$
\begin{aligned}
& \left\|F\left(x_{1}, x_{2}\right)-F^{\prime}\left(x_{1}, x_{2}\right)\right\| \\
& =\left\|\frac{1}{4^{n}} F\left(2^{n} x_{1}, 2^{n} x_{2}\right)-\frac{1}{4^{n}} F^{\prime}\left(2^{n} x_{1}, 2^{n} x_{2}\right)\right\| \\
& \leq\left\|\frac{1}{4^{n}} F\left(2^{n} x_{1}, 2^{n} x_{2}\right)-\frac{1}{4^{n}} f\left(2^{n} x_{1}, 2^{n} x_{2}\right)\right\|
\end{aligned}
$$


$+\left\|\frac{1}{4^{n}} F^{\prime}\left(2^{n} x_{1}, 2^{n} x_{2}\right)-\frac{1}{4^{n}} f\left(2^{n} x_{1}, 2^{n} x_{2}\right)\right\|$

$\leq \frac{2}{4^{n}} \frac{25}{3} \delta$

whence letting $n \rightarrow \infty$ we obtain $F=F^{\prime}$.

\section{Acknowledgements}

Lin Wang was supported by Promotive research fund for excellent young and middle-aged scientists of Shandong Province, China, by Hall of Shandong Province Science and Technology (No. BS2013SF014). Peisheng Ji is corresponding author.

\section{References}

[1] S.M. Ulam: A Collection of Mathematical Problems. (Interscience Press, New York 1960).

[2] D.H. Hyers: Proc. Natl. Acad. Sci. USA. Vol. 27 (1941), p. 222

[3] Th.M. Rassias: Proc. Amer. Math. Soc. Vol. 72 (1978), p. 297

[4] C. Park, J.R. Lee: The Austr. J. Math. Anal. Appl. Vol. 18(1) (2011), p. 1

[5] Soon-Mo Jung: Hyers-Ulam-Rassias stability of functional equations in Nonlinear Analysis. (Springer Optimization and its applications, Korea 2011).

[6] K. Ciepliński: Appl. Math. Lett. Vol. 23 (2010), p. 1291

[7] F.Skof: On the stability on functional equations on a restricted domain and a related topic. In 'Stability of Mappings of Hyers-Ulam Type' (edited by Th. M. Rassias and J. Tabor: Hadronic Press, Palm Harbor, Florida 1994), p. 141

[8] S.-M. Jung: J. Math. Anal. Appl. Vol. 222 (1998), p. 126

[9] Bogdan Batko and Jacek Tabor: Aequationes Math. Vol. 57 (1999), p.221

[10] Yang-Hi Lee: Int. Journal of Math. Analysis. Vol. 7(55) (2013), p.2745 